\section{The Natural History of Type 2 Endoleaks after EVAR Justifies Conservative Management}

\section{Abstract}

Objective: Management of type 2 endoleaks (T2LS) after endovascular aneurysm repair (EVAR) has been controversial. Some advocate for conservative management, while others believe that intervention is indicated. This study investigated the natural history of T2Ls in order to derive direction in management.

Methods: Patients who had EVAR at the Long Beach VA were retrospectively identified and computerized tomographic angiography (CTA) was independently reviewed by a radiologist and a vascular surgeon. T2Ls were analyzed for the following outcomes: rupture, duration of endoleak, spontaneous resolution, changes in the size of the aneurysm sac, and reintervention rates.

Results: Of the 160 patients who had completed required followup to date (mean 3 years) after EVAR, 39 patients (24.4\%) were identified as having a T2L on CTA imaging. 6 of these 39 patients $(15.4 \%)$ required repair due to aneurysm sac growth $>1 \mathrm{~cm}$. Two (5.13\%) were repaired with an open procedure and $4(10.3 \%)$ with an endovascular approach. Of these 6 aneurysm leaks requiring repair, 4 $(66.7 \%)$ had a simultaneous endoleak (types 1 or 3 ) in addition to the identified T2L. Spontaneous resolution of T2Ls occurred in 16 (41.0\%) patients.

Conclusions: Overall, we found that $85 \%$ of patients who had $\mathrm{T} 2 \mathrm{LS}$ did not require intervention after a mean follow up time of 3 years. The association of a type 1 or 3 endoleak with a $\mathrm{T} 2 \mathrm{~L}$ was more likely to require correction due to aneurysm expansion $>1 \mathrm{~cm}$, thus T2Ls associated with another type of endoleak require more aggressive management.

Keywords: Type 2 endoleaks; Endovascular aneurysm repair; Endoleak management; Endoleak natural history

\section{Mark Ajalata*, Russell A Williamsa and Samuel E Wilsona}

\author{
Department of Vascular Surgery, Long \\ Beach VA Medical Center, Institution, \\ 5901 E 7th Street, Long Beach, CA 90822, \\ Canada
}

*Corresponding author: Mark Ajalat

\section{Ajalat.Mark@gmail.com}

Department of Vascular Surgery, 1331 Journeys End Drive, La Canada, CA 91011, Canada.

Tel: 6262901977

Fax: 8187902046

Citation: Ajalata M, Williamsa RA, Wilsona SE (2017) The Natural History of Type 2 Endoleaks after EVAR Justifies Conservative Management. J Vasc Endovasc Surg. Vol.2 No.4:34

Received: October 12, 2017; Accepted: November 05, 2017; Published: November 13, 2017

\section{Introduction}

Open repair of abdominal aortic aneurysms (AAAs) has been largely replaced by endovascular aneurysm repair (EVAR), which is a less invasive and less morbid procedure [1,2]. Recent studies have documented the incidence of EVAR, which accounted for only $5.2 \%$ of AAA repairs in 2000 , but by 2010 had increased to $74 \%$ of repairs $[3,4]$. Presently, only a minority of patients will undergo open repair, mostly due to adverse anatomy $[3,4]$. This shift in approach of AAA repair to endovascular technique has been justified by the lower operative mortality and fewer severe postoperative complications $[1,2,5]$. Although EVAR has generally been adopted as a safer approach, type 2 endoleaks
(T2Ls) are a commonly recognized postoperative complication. Their significance, however, has been widely debated. T2Ls occur when blood flows in a retrograde manner from an aortic branch vessel, usually a lumbar artery or the inferior mesenteric artery, into the aneurysm sac [6]. Optimal management of T2Ls is controversial due to conflicting data. Some surgeons believe that T2Ls are likely to lead to aneurysm expansion and rupture, and hence require close surveillance and early reintervention [7]. Other surgeons believe that T2Ls have a benign course with spontaneous resolution and low aneurysm rupture rate, thus indicating a more conservative approach [6]. The overall goal of this study is to document the natural history of T2Ls using data from a single center in order to derive direction in management. 


\section{Patients and Methods}

The Veterans Affairs Long Beach Healthcare System is a diverse institution providing comprehensive inpatient and outpatient care to over 50,000 veterans [8]. The data collected for this retrospective study was compiled using the medical center's computerized patient record system (CPRS). Data acquisition for this retrospective study was performed in a secure manner with de-identified patient data and exclusion of all protected health information, hence our study is not subject to patient consent. Data was analyzed from 4 IRB approved aneurysm studies (1001, 1195, 979, 1345).

We obtained complete medical records on 175 patients who underwent an EVAR procedure for aneurysm repair. Of these, 15 patients were excluded due to lack of scheduled postoperative computed tomography angiography (CTA), yielding a total of 160 patients for review. The presence of endoleaks (type 1, 2, or 3) among these patients was identified independently by a radiologist and a member of the vascular surgery team. EVAR was performed with the following stent grafts: Anaconda (Vascutek, Terumo, Inchinnan, Scotland), Excluder (W.L. Gore \& Associates, Flagstaff, AZ, USA), Power Link System (Endologix, Irvine, CA, USA), Endurant (Medtronic Vascular, Santa Rosa, CA, USA), and Pythagorus (Lombard, Oxfordshire, UK).

Patients were followed using CTA with a three-phase intravenous contrast protocol using $1 \mathrm{~mm}$ slices at 30 days, 6 months, and annually for 5 years. Helical images were taken along with intravenous administration of contrast material in order to allow for dynamic and late-phase assessment of the aorta. The maximal aneurysm diameter was measured with electronic calipers from outer wall to outer wall. The following outcomes for the identified T2Ls were analyzed: rupture, duration of endoleak, spontaneous resolution, changes in the size of the aneurysm sac, and reintervention rates. Spontaneous resolution was defined as a $T 2 L$ that resolved over time without intervention as evidenced by follow-up CTA. Persistent endoleaks were defined as T2Ls that lasted 6 months or longer. Aneurysm sac growth was defined as $>5 \mathrm{~mm}$ maximal aneurysm diameter change when comparing the earliest postoperative CTA (usually the 1 month follow-up) with the CTA demonstrating the largest postoperative aneurysm diameter. Any delayed endoleaks were also documented by the appearance of a T2L after the first postoperative CTA appeared normal. We only included those type 1 and 3 endoleaks that were discovered on further investigation after identifying the $T 2 \mathrm{~L}$ on CTA.

\section{Results}

160 patients had completed the required follow-up as of May 2015 (mean 3 years) after EVAR. Of these patients, 39 (24.4\%) were identified as having a T2L on CTA imaging (Table 1). In the fifth year after EVAR, one patient $(0.63 \%)$, who did not have any type 1 or 2 endoleak died of a ruptured aneurysm after $0.9 \mathrm{~cm}$ expansion. Of the 39 patients, $6(15.4 \%)$ required repair due to aneurysm sac growth greater than $1 \mathrm{~cm}$. Two $(5.13 \%)$ were repaired with an open procedure and 4 (10.3\%) with an endovascular approach. Of these 6 aneurysms requiring repair, $4(66.7 \%)$ had a simultaneous type 1 or 3 endoleak in addition to the identified T2L.

Spontaneous resolution of T2L occurred in 16 (41.0\%) patients and persistent endoleaks lasting longer than 6 months occurred in 22 (56.4\%). Four patients $(10.3 \%)$ had delayed T2Ls that presented $4,9,12$, and 23 months after their 30 day postoperative CT was normal. None of the 4 patients with delayed T2Ls required reintervention and none had aneurysm sac growth greater than $5 \mathrm{~mm}$.

Of the 39 patients with T2Ls, 8 (20.5\%) were associated with an additional type 1 or 3 endoleak which were discovered after identification of the initial T2Ls. 4 of these have had intervention due to aneurysm expansion greater than $1 \mathrm{~cm}$, and in the remaining 4, correction is pending. Among the 31 patients $(79.5 \%)$ with isolated T2Ls, 8 (25.8\%) had an aneurysm sac enlargement of $5 \mathrm{~mm}$ to $1 \mathrm{~cm}$.

\section{Discussion}

The incidence of T2Ls cited in the literature varies between $8 \%-45 \%$ [9]. We believe that this variation is a consequence of diagnostic methods (ultrasound versus CTA) and observer interpretation. Nevertheless, our incidence of $24.4 \%$ is within the commonly accepted variance. Although several treatment strategies exist for repair of T2Ls after EVAR (injection of thrombogenic materials into the aortic sac, transarterial embolization, or open repair), optimal management of this postoperative complication is controversial and the results of intervention are often disappointing $[10,11]$. Current data is conflicting, and it has not been determined if conservative management or selective or aggressive intervention yields better outcomes [11,12]. Some authors claim that T2Ls follow a benign natural course due to the rarity of aneurysm sac expansion and rupture (Table 2). Others have proposed that early reintervention is beneficial due to successful prevention of the late complications of sac expansion (Table 3).

Table 1 Outcome of Type 2 Endoleaks - ${ }^{a} 4$ patients had delayed T2Ls 4, 9, 12, and 23 months after normal post op CT. None required reintervention or had aneurysm sac growth greater than $5 \mathrm{~mm}$.

\begin{tabular}{c|c|c|c}
\hline & Type 2 Endoleaks & Number & Outcome \\
\hline $\begin{array}{c}\text { Type 2 Endoleaks } \\
\text { Requiring Repair }\end{array}$ & $\begin{array}{c}\text { Inferior Mesenteric Artery } \\
\text { Lumar Arteries (with associated } \\
\text { type 1 or 3 endoleaks) }\end{array}$ & 2 & Both resolved by embolization, one with coils and the other with onyx \\
$\begin{array}{c}\text { Type 2 Endoleaks Without } \\
\text { Intervention } \\
\text { Total }\end{array}$ & $\begin{array}{c}\text { Spontaneous Resolution } \\
\text { a Unresolved }\end{array}$ & 16 & 2 resolved with open repair, 2 resolved with endovascular repair \\
\hline
\end{tabular}


Table 2 Clinical Reports on Conservative Management of Type 2 Endoleaks.

\begin{tabular}{|c|c|c|c|c|}
\hline $\begin{array}{c}\text { Author (Year), Type } \\
\text { of Study }\end{array}$ & $\begin{array}{c}\text { Number of Patients } \\
\text { with EVAR }\end{array}$ & $\%$ of T2Ls & $\begin{array}{l}\text { Follow-up Period } \\
\text { (months) }\end{array}$ & Significant Results \\
\hline $\begin{array}{l}\text { Nolz et al. (2012) } \\
\text { [14]. Retrospective } \\
\text { Analysis }\end{array}$ & 407 & $12.0 \%$ (49 patients) & $68.1 \pm 23.8$ & $\begin{array}{l}\text { Persistent T2Ls lead to significant sac enlargement when } \\
\text { compared with aneurysms lacking T2Ls. Despite this significant } \\
\text { enlargement, there was no increase in rupture or mortality } \\
\text { rates. Persistent T2Ls with aneurysm growth require close } \\
\text { surveillance, but intervention is not indicated. }\end{array}$ \\
\hline $\begin{array}{l}\text { Sidloff et al. (2014) } \\
\text { [10]. Prospective } \\
\text { Analysis }\end{array}$ & 904 & $\begin{array}{c}19.4 \% \text { (175 } \\
\text { patients) }\end{array}$ & 43.2 & $\begin{array}{l}\text { By } 6 \text { months, } 54 \% \text { of T2Ls spontaneously resolved. Those with } \\
\text { T2Ls had a significantly higher survival. Patients with isolated } \\
\text { T2Ls have equivalent aneurysm related mortality when } \\
\text { compared to those without T2Ls. Conservative approach is } \\
\text { safe. }\end{array}$ \\
\hline $\begin{array}{l}\text { Rayt et al. (2009) } \\
\text { [15]. Retrospective- } \\
\text { Prospective Study }\end{array}$ & 369 & $11.1 \%$ (41 patients) & 48 & $\begin{array}{l}48 \% \text { of T2Ls spontaneously resolved. } 20 \% \text { had an enlarging } \\
\text { sac. No reported aneurysm related deaths, and no open } \\
\text { repairs were required. }\end{array}$ \\
\hline
\end{tabular}

Abbreviations: EVAR - endovascular aneurysm repair; T2L - type 2 endoleak; AAA - abdominal aortic aneurysm.

Table 3 Clinical Reports Advocating for Intervention Among Type 2 Endoleaks.

\begin{tabular}{|c|c|c|c|c|c|}
\hline $\begin{array}{c}\text { Author (Year), Type of } \\
\text { Study }\end{array}$ & $\begin{array}{c}\text { Number of Patients } \\
\text { with EVAR }\end{array}$ & $\%$ of T2Ls & $\begin{array}{l}\text { Number of } \\
\text { Intervention }\end{array}$ & $\begin{array}{c}\text { Follow-up } \\
\text { Period (months) }\end{array}$ & Significant Results \\
\hline $\begin{array}{l}\text { Fabre et al. (2015) [19]. } \\
\text { Retrospective Analysis }\end{array}$ & 187 & NS & $83(44.7 \%)$ & $24 \pm 11$ & $\begin{array}{l}\text { Coil embolization in those at risk for } \mathrm{T} 2 \mathrm{~L} \\
\text { during EVAR is fast and effective with few } \\
\text { complications. }\end{array}$ \\
\hline $\begin{array}{l}\text { Batti et al. (2013) [18]. } \\
\text { Retrospective analysis }\end{array}$ & 700 & $\begin{array}{c}28.9 \%(201 \\
\text { patients) }\end{array}$ & $\begin{array}{l}40 \text { (19.9\% of } \\
\text { patients with } \\
\text { T2Ls) }\end{array}$ & 31.3 & $\begin{array}{l}\text { T2Ls are associated with aneurysm sac } \\
\text { enlargement, and persistent T2Ls are the } \\
\text { most dangerous, leading to life-threatening } \\
\text { complications. Early and specific treatment is } \\
\text { recommended. }\end{array}$ \\
\hline $\begin{array}{l}\text { Sarac et al. (2012) [20]. } \\
\text { Retrospective analysis }\end{array}$ & 809 & NS & 95 (11.7\%) & NS & $\begin{array}{l}\text { Secondary intervention through embolization } \\
\text { of T2Ls is successful early on in preventing } \\
\text { further aneurysm sac growth and rupture. } \\
\text { Patients with greater risk factors (chronic } \\
\text { smokers/ hyperlipidemia) may require multiple } \\
\text { procedures and require long-term surveillance. }\end{array}$ \\
\hline $\begin{array}{l}\text { Van Marrewijk } \\
\text { et al. (2004) [17]. } \\
\text { Retrospective Analysis }\end{array}$ & 3595 & $\begin{array}{l}8.90 \% \text { ( } 320 \\
\text { patients) }\end{array}$ & NS & 15 & $\begin{array}{l}\text { T2Ls are not benign and are associated with } \\
\text { more frequent reinterventions and aneurysm } \\
\text { sac enlargement. Patients with T2Ls require } \\
\text { more frequent surveillance, and aneurysm } \\
\text { expansion indicates reintervention. }\end{array}$ \\
\hline $\begin{array}{l}\text { Jones et al. (2007) [7]. } \\
\text { Retrospective Analysis }\end{array}$ & 873 & $\begin{array}{c}18.9 \%(164 \\
\text { patients) }\end{array}$ & NS & 32.6 & $\begin{array}{l}\text { Persistent T2LS }>6 \text { months were associated } \\
\text { with a higher incidence of clinically adverse } \\
\text { outcomes such as aneurysm sac growth, } \\
\text { conversion to open repair, and risk of rupture. } \\
\text { T2Ls lasting longer than } 6 \text { months require } \\
\text { aggressive management and reintervention. }\end{array}$ \\
\hline
\end{tabular}

Abbreviations: NS - not stated.

Support for conservative management of T2LS is based on the relatively high percentage of $\mathrm{T} 2 \mathrm{Ls}$ that resolve spontaneously (Table 2). Gelfand et al. advised that T2Ls should be followed carefully, observing that $58 \%$ of 2 Ls should resolve spontaneously within a 12-month time period [13]. In addition, they posited that T2Ls had no increase risk for aneurysm rupture if their guidelines 
were followed [13]. Their guidelines for intervention, now a decade old, included the following: a symptomatic or pulsatile AAA sac, sac enlargement of $>5 \mathrm{~mm}$, or an aneurysmal sac pressure $>20 \%$ of systolic pressure [13]. Similarly, Sidloff et al. found that by 6 months, 54\% of T2Ls spontaneously resolved and aneurysm related mortality was similar to those patients who did not present with T2Ls [10]. Surprisingly, they found that the 175 patients with T2Ls actually demonstrated a significantly higher survival rate than those without endoleaks, perhaps because surveillance lead to closer follow up of their general medical condition [10]. Although supporters of conservative management claim T2Ls behave clinically benign, they concede that T2Ls may contribute to aneurysm sac enlargement [13-15]. But, despite the fact that persistent leaks may lead to sac enlargement, they have also found no increase in rupture or mortality rates when compared to those without T2Ls (Table 2) [13-16]. Overall, these authors believe that a conservative approach is safe, and with few exceptions, intervention is not indicated in the treatment of T2Ls.

On the other hand, clinical reports have suggested that T2Ls are associated with aneurysm related adverse events which demand aggressive management and reintervention (Table 3 ). Jones et al. found that persistent endoleaks greater than 6 months duration had a greater incidence of aneurysm sac growth, conversion to open repair, and risk of rupture [7]. Marrewijk et al. similarly claimed that T2Ls are not benign, and suggested that aneurysm expansion $>8 \mathrm{~mm}$ is an indication for reintervention [17]. A retrospective analysis of 700 patients undergoing EVAR also demonstrated how T2Ls may not always have a benign course [18]. In this study, Batti et al. found that patients with T2Ls had significantly greater aneurysm sac enlargement $>5 \mathrm{~mm}$ and were associated with more complications such as death, rupture, and reintervention [18]. Based on their results, they concluded the following regarding the management of T2Ls: 1) Intervention to treat the endoleak if the aneurysm enlarges 2) Repeat CT scan every 6 months then yearly thereafter if the sac size is stable with unresolved T2L [18].

Although there are several different approaches for repair of T2Ls after EVAR, many support the use of coil embolization as an effective treatment to prevent late complications of T2Ls [19].

Secondary intervention through embolization has shown to be effective in preventing aneurysm sac enlargement and rupture within a year after the procedure [20]. However, some of these patients when followed up in the long term were found to have late AAA expansion after embolization, leading Sarac et al. to conclude that coil embolization alone may not be the best procedure for long term efficacy [20]. Baum et al. recommend direct translumbar endoleak embolization preferentially over transarterial endoleak embolization after $\mathrm{T} 2 \mathrm{~L}$ is confirmed by angiography [21].

T2Ls may result from a patent inferior mesenteric artery (IMA) which receives retrograde filling from the superior mesenteric artery (SMA) via the marginal artery of Drummond or the arc of Riolan, further pressurizing the aneurysm sac (Figure 1A) [22]. Guntner et al. concluded that there was a significantly higher incidence of $\mathrm{T} 2 \mathrm{LS}$ in patients with greater cross sectional area of the IMA ostium at the aortic root [23]. Knowledge of this collateralization between the SMA and IMA in T2Ls has guided the surgical approach of coil embolization in successfully preventing and treating this complication (Figure 1B) $[24,25]$. Although there were only 2 patients in this study whose T2Ls demonstrated this type of collateralization, both were successfully treated with coil embolization.
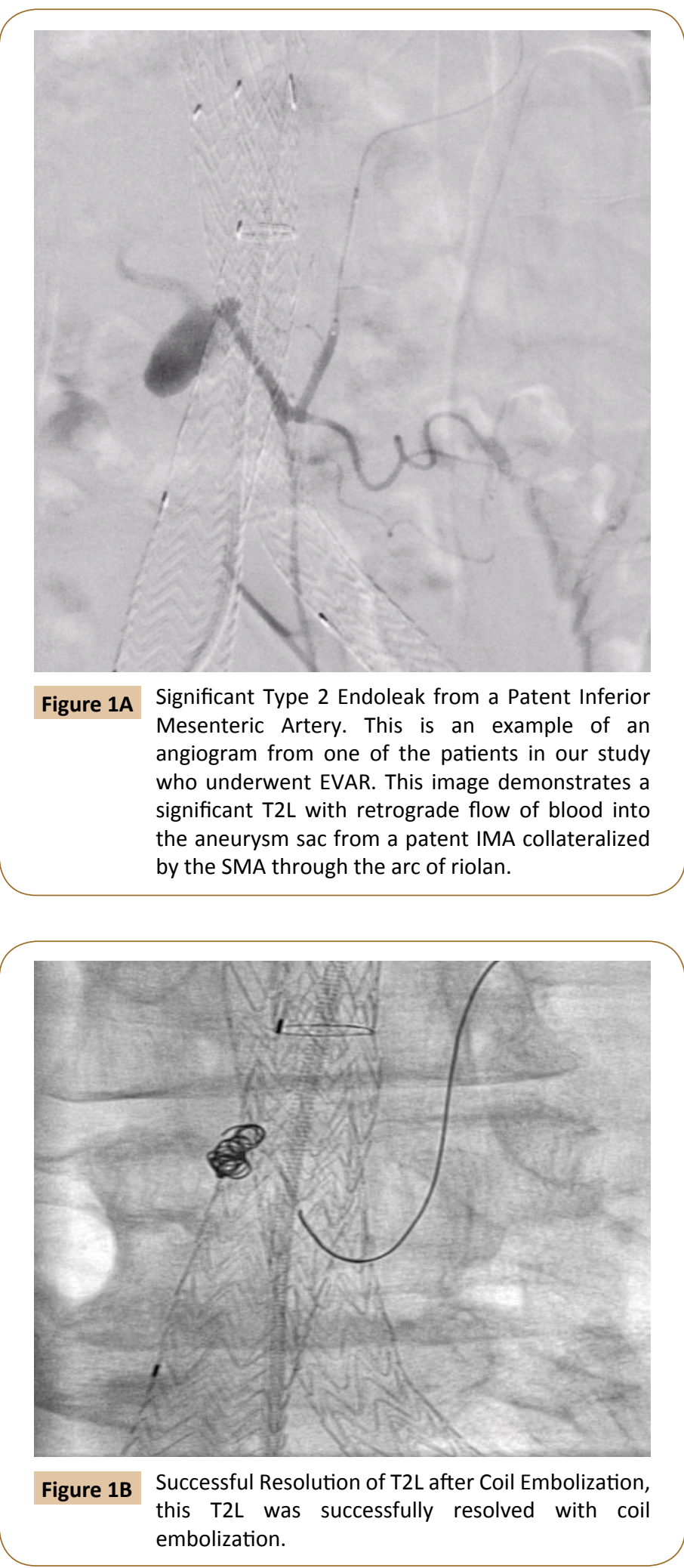
Our findings in this study continue to support the position that T2Ls are largely benign postoperative complications which require close surveillance, but not aggressive intervention. Although there was one aneurysm related mortality in this study, there were no recognized endoleaks associated with the patient's graft. Overall we found that $15.4 \%$ of patients that presented with T2Ls (either in isolation or simultaneously with another type of endoleak) required reintervention with open repair or an endovascular approach due to an enlarging aneurysm sac $>1 \mathrm{~cm}$. More importantly, 2/3 of these T2Ls which required reintervention were later found to be associated with simultaneous type 1 or 3 endoleaks. Currently there is no debate regarding the clinical management of type 1 or 3 endoleaks, as it is common practice to pursue intervention when discovered. In patients in this study who were ultimately identified as having simultaneous T2Ls and type 1 endoleaks, recognition of the T2L in follow up studies lead to the discovery of the type 1 endoleak. Having an additional type of endoleak simultaneously with a $\mathrm{T} 2 \mathrm{~L}$ increases the risk for sac enlargement and reintervention. On the other hand, we found that $25.8 \%$ of isolated T2Ls had an enlarging aneurysm sac of greater than $5 \mathrm{~mm}$, but none of these required reintervention. Given these findings, we recommend conservative management and close surveillance of T2Ls that occur in isolation, given their relatively benign natural history. In addition, we recommend more aggressive intervention when a simultaneous type 1 or 3 endoleak is present, aneurysm expansion is greater than $1 \mathrm{~cm}$ in diameter, and in the special

\section{References}

1 Prinssen M, Verhoeven EL, Buth J, Cuypers PW, van Sambeek $\mathrm{MR}$, et al. (2004) A randomized trial comparing conventional and endovascular repair of abdominal aortic aneurysms. N Engl J Med 351:1607-1618.

2 Wickremesekera J, Farmillo W, Hawkins S, Zargar H, Choudhary A, et al. (2007) Endoluminal repair of abdominal aortic aneurysm: the Middlemore Hospital experience. N Z Med J.

3 Dua A, Kuy S, Lee CJ, Upchurch GR, Desai SS (2014) Epidemiology of aortic aneurysm repair in the United States from 2000 to 2010. J Vasc Surg 59: 1512-1517.

4 Rose J, Evans C, Barleben A, Bandyk D, Wilson SE, et al. (2014) Comparative safety of endovascular aortic aneurysm repair over open repair using patient safety indicators during adoption. JAMA Surg 149:926-932.

5 Siracuse JJ, Gill HL, Graham AR, Schneider DB, Connolly PH, et al. (2014) Comparative safety of endovascular and open surgical repair of abdominal aortic aneurysms in low-risk male patients. J Vasc Surg 60: 1154-1158.

6 Ricotta JJ (2010) Endoleak management and postoperative surveillance following endovascular repair of thoracic aortic aneurysms. J Vasc Surg 52: 91S-99S.

7 Jones JE, Atkins MD, Brewster DC, Chung TK, Kwolek CJ, et al. (2007) Persistent type 2 endoleak after endovascular repair of abdominal aortic aneurysm is associated with adverse late outcomes. J Vasc Surg 46: 1-8. case of direct collateralization of the inferior mesenteric artery from the superior mesenteric artery.

Our findings are consistent with Rayt et al., because although $56.4 \%$ of endoleaks persisted longer than 6 months, $41 \%$ of T2Ls spontaneously resolved. Moreover, we found that having a delayed $\mathrm{T} 2 \mathrm{~L}$ does not lead to further downstream adverse consequences, as none of the delayed endoleaks required additional treatment or had enlarging aneurysm sacs $>5 \mathrm{~mm}$. Although $10.3 \%$ of T2Ls occurred between 4 and 23 months after surgery, these patients had similar outcomes when compared to those who developed T2Ls either intraoperatively or by the 30 day post-operative CT. Therefore we recommend a similar conservative management, with close observation of delayed T2Ls.

\section{Conclusion}

Overall, we found that $85 \%$ of patients who had T2Ls did not require intervention after a mean follow up of 3 years. The one aneurysm related mortality in our 160 patients did not have any identifiable endoleak. The association of a type 1 or 3 endoleak with a $\mathrm{T} 2 \mathrm{~L}$ was more likely to require correction due to aneurysm expansion $>1 \mathrm{~cm}$. T2Ls associated with another type of endoleak require more aggressive management. We conclude that there are 3 circumstances in which we recommend consideration for reintervention: 1) T2L associated with either a type 1 or 3 endoleak 2) Greater than $1 \mathrm{~cm}$ aneurysm sac enlargement 3) The finding of a collateralized inferior mesenteric artery feeding vessel.

8 US Department of Veterans Affairs (2016) VA Long Beach Healthcare System.

9 Silverberg D, Baril DT, Ellozy SH, Carroccio A, Greyrose SE, et al. (2006) An 8-year experience with type II endoleaks: natural history suggests selective intervention is a safe approach. J Vasc Surg 44: 453-459.

10 Sidloff DA, Gokani V, Stather PW, Choke E, Bown MJ, et al. (2014) Editor's Choice-Type II Endoleak: Conservative Management Is a Safe Strategy. Eur J Vasc Endovasc Surg 48: 391-399.

11 Hajibandeh S, Ahmad N, Antoniou GA, Torella F (2015) Is intervention better than surveillance in patients with type 2 endoleak postendovascular abdominal aortic aneurysm repair? Interact Cardiovasc Thorac Surg 20: 128-134.

12 Karthikesalingam A, Thrumurthy SG, Jackson D, Choke E, Sayers $\mathrm{RD}$, et al. (2012) Current evidence is insufficient to define an optimal threshold for intervention in isolated type II endoleak after endovascular aneurysm repair. J Endovasc Ther 19: 200-208.

13 Gelfand DV, White GH, Wilson SE (2006) Clinical significance of type II endoleak after endovascular repair of abdominal aortic aneurysm. Ann Vasc Surg 20: 69-74.

14 Nolz R, Teufelsbauer H, Asenbaum U, Beitzke D, Funovics M, et al. (2012) Type II endoleaks after endovascular repair of abdominal aortic aneurysms: fate of the aneurysm sac and neck changes during long-term follow-up. J Endovasc Ther 19: 193-199.

15 Rayt HS, Sandford RM, Salem M, Bown MJ, London NJ, et al. (2009) Conservative management of type 2 endoleaks is not associated 
with increased risk of aneurysm rupture. Eur J Vasc Endovasc Surg 38: 718-723.

16 Walker J, Tucker LY, Goodney P, Candell L, Hua H, et al. (2015) Type II endoleak with or without intervention after endovascular aortic aneurysm repair does not change aneurysm-related outcomes despite sac growth. J Vasc Surg 62: 551-561.

17 Van Marrewijk CJ, Fransen G, Laheij RJ, Harris PL, Buth J (2004) Is a type II endoleak after EVAR a harbinger of risk? Causes and outcome of open conversion and aneurysm rupture during follow-up. Eur J Vasc Endovasc Surg 27: 128-137.

18 El Batti S, Cochennec F, Roudot-Thoraval F, Becquemin JP (2013) Type II endoleaks after endovascular repair of abdominal aortic aneurysm are not always a benign condition. J Vasc Surg 57: 1291-1297.

19 Fabre D, Fadel E, Brenot P, Hamdi S, Caro AG, et al. (2015) Type II endoleak prevention with coil embolization during endovascular aneurysm repair in high-risk patients. J Vasc Surg 62: 1-7.

20 Sarac TP, Gibbons C, Vargas L, Liu J, Srivastava S, et al. (2012) Longterm follow-up of type II endoleak embolization reveals the need for close surveillance. J Vasc Surg 55: 33-40.

21 Baum RA, Carpenter JP, Golden MA, Velazquez OC, Clark TW, et al. (2002) Treatment of type 2 endoleaks after endovascular repair of abdominal aortic aneurysms: comparison of transarterial and translumbar techniques. J Vasc Surg 35: 23-29.

22 Velazquez OC, Baum RA, Carpenter JP, Golden MA, Cohn M, et al. (2000) Relationship between preoperative patency of the inferior mesenteric artery and subsequent occurrence of type II endoleak in patients undergoing endovascular repair of abdominal aortic aneurysms. J Vasc Surg 32: 777-788.

23 Güntner O, Zeman F, Wohlgemuth WA, Heiss P, Michael Jung E, et al. (2013) Inferior mesenteric arterial type II endoleaks after endovascular repair of abdominal aortic aneurysm: are they predictable?. Radiol 270: 910-919.

24 Axelrod DJ, Lookstein RA, Guller J, Nowakowski FS, Ellozy S, et al. (2004) Inferior mesenteric artery embolization before endovascular aneurysm repair: technique and initial results. J Vasc Interv Radiol 15: 1263-1267.

25 Sucandy I, Kim H, Sullivan TR (2011) Endovascular management of the patent inferior mesenteric artery in two cases of uncontrolled type II endoleak after endovascular aneurysm repair. N Am J Med Sci 3: 387. 